\title{
NEW COMPRESSED SENSING ISAR IMAGING ALGORITHM BASED ON LOG-SUM MINIMIZATION
}

\author{
Cheng Ping * Z Zhao Jiaqun ${ }^{* *}$
}

\begin{abstract}
To improve the performance of inverse synthetic aperture radar (ISAR) imaging based on compressed sensing (CS), a new algorithm based on log-sum minimization is proposed. A new interpretation of the algorithm is also provided. Compared with the conventional algorithm, the new algorithm can recover signals based on fewer measurements, in looser sparsity condition, with smaller recovery error, and it has obtained better sinusoidal signal spectrum and imaging result for real ISAR data. Therefore, the proposed algorithm is a promising imaging algorithm in CS ISAR.
\end{abstract}

K e y w or d s: compressed sensing (CS), inverse synthetic aperture radar (ISAR), log-sum

\section{INTRODUCTION}

Inverse synthetic aperture radar (ISAR) can supply high resolution images of targets all day, all-weather and over long distance, so it has found wide applications in military and for civilian use. The actual demands of antiinterference, maneuvering target imaging and super resolution imaging promote the research of ISAR imaging using limited pulses $[1,2]$. Compressed sensing (CS), a big idea in signal processing field, can reconstruct signals based on limited measurements by using the sparsity of signals [3]. As ISAR targets can be described by a few strong scattering centers, which is consistent with the sparsity requirement of CS, applying CS into ISAR imaging has great potential.

To get good CS ISAR imaging performance, proper sparsity penalty functions should be used. At present, CS ISAR imaging are generally based on $l_{1}$ norm to approximate $l_{0}$ norm. But geometrically, $l_{1}$ norm can't approximate the desired $l_{0}$ norm effectively. A weighted formulation of $l_{1}$ minimization has been designed. However it was proposed instinctively [4]. Also Gini index was used to measure the sparsity of signal in CS ISAR. But theoretical conditions need to be established to guarantee exact reconstruction [5]. In 2012, Zhao et al proposed a novel reconstruction model based on Meridian norm [6]. It is a pity that the solution algorithm is complex. In $2013, \mathrm{Li}$ et al applied smoothed $l_{0}$ norm in CS ISAR imaging [7]. However, it often requires a large number of iterations and cannot perform equally well in all scenarios [8].

To improve ISAR images quality based on CS, an imaging algorithm based on log-sum minimization is proposed in the paper. Compared with $l_{1}$ norm, log-sum penalty function can approximate $l_{0}$ norm better $[9,10]$. Although the optimization problem based on log-sum penalty function is non-convex, it can be solved effectively by majorization-minimization (MM) algorithm
[11]. Compared with the existing methods, the proposed algorithm has three advantages. The first advantage is that it is simple and has low computational cost. The second is that it has good theoretical basis. The third is that it can get good recovery performance.

\section{ISAR SIGNAL MODEL}

ISAR transmits electromagnetic pulses and processes the returned pulses to image targets. Linear frequency modulated (LFM) signal transmitted by radar can be expressed

$$
s(t, \tau)=\operatorname{rect} \frac{t}{T_{p}} \exp \left(j 2 \pi\left(f_{c} t+\frac{1}{2} \mu t^{2}\right)\right)
$$

where $t$ is fast time, $\tau$ is slow time, $T_{p}$ is pulse width, $f_{c}$ is carrier frequency, $\mu$ is chirp rate, and $\operatorname{rect}(\cdot)$ is rectangle function. The received signal from scatter $P$ can be written as

$$
\begin{array}{r}
s_{r}(t, \tau)=\sigma \operatorname{rect} \frac{t-2 R(\tau) / c}{T_{p}} \exp \left(j 2 \pi \left(f_{c}\left(t-\frac{2 R(\tau)}{c}\right)\right.\right. \\
\left.\left.+\frac{1}{2} \mu\left(t-\frac{2 R(\tau)}{c}\right)^{2}\right)\right)
\end{array}
$$

where $\sigma$ is reflection coefficient of scatter $P, R(\tau)$ is distance between scatter $P$ and radar, and $c$ is light speed. The reference signal is

$$
\begin{array}{r}
s_{\mathrm{ref}}(t, \tau)=\operatorname{rect} \frac{t-2 R_{\mathrm{ref}}(\tau) / c}{T_{\mathrm{ref}}} \\
\exp \left(j 2 \pi \left(f_{c}\left(t-\frac{2 R_{\mathrm{ref}}(\tau)}{c}\right)\right.\right. \\
\left.\left.+\frac{1}{2} \mu\left(t-\frac{2 R_{\mathrm{ref}}(\tau)}{c}\right)^{2}\right)\right)
\end{array}
$$

\footnotetext{
* College of Computer and Information, Hohai University, 211100 Nanjing, China, chengping1219@163.com; ${ }^{* *}$ College of Science, Hohai University, 210098 Nanjing, China, jqzhao_03@163.com
} 
where $R_{\text {ref }}(\tau)$ is distance between reference scatter $Q$ and radar, and $T_{\text {ref }}$ is pulse width of reference signal. After dechirping, the echo signal becomes

$$
\begin{aligned}
s_{d e}(t, \tau)= & s_{r}(t, \tau) \times s_{\mathrm{ref}}^{*}(t, \tau)=\sigma \times \operatorname{rect} \frac{t-2 R(\tau) / c}{T_{p}} \\
& \times \exp \left(-j \frac{4 \pi}{c} \mu\left(t-\frac{2 R_{\mathrm{ref}}(\tau)}{c}\right) R_{\Delta}(\tau)\right) \\
& \quad \times \exp \left(-j \frac{4 \pi}{c} f_{c} R_{\Delta}(\tau)+j \frac{4 \pi \mu}{c^{2}} R_{\Delta}^{2}(\tau)\right)
\end{aligned}
$$

where ${ }^{*}$ is conjugation operator, and $R_{\Delta}(\tau)=R(\tau)-$ $R_{\text {ref }}(\tau)$. Applying Fourier transform to formula (4) with respect to fast timet, the following is obtained

$$
\begin{aligned}
S_{d e}(f, \tau)= & \sigma T_{p} \operatorname{sinc}\left(T_{p}\left(f+\frac{2 \mu}{c} R_{\Delta}(\tau)\right)\right. \\
& \times \exp \left(-j \frac{4 \pi}{c} R_{\Delta}(\tau)\left(f_{c}+f-\frac{\mu}{c} R_{\Delta}(\tau)\right)\right)
\end{aligned}
$$

where $f$ is range frequency. After eliminating the residual video phase (RVP), the following is obtained

$$
\begin{aligned}
& S_{d e}^{\prime}(f, \tau)= \\
& \quad \sigma T_{p} \operatorname{sinc}\left(T_{p}\left(f+\frac{2 \mu}{c} R_{\Delta}(\tau)\right)\right) \exp \left(-j \frac{4 \pi}{c} R_{\Delta}(\tau) f_{c}\right) .
\end{aligned}
$$

It can be seen from (6) that the peak locates at $f$ equals $-\frac{2 \mu}{c} R_{\Delta}(\tau)$. If the sinc function in (6) is approximated by Dirac delta function, formula (6) can be rewritten as

$$
S_{d e}^{\prime}(f, \tau) \approx \sigma T_{p} \delta\left(f+\frac{2 \mu}{c} R_{\Delta}(\tau)\right) \exp \left(-j \frac{4 \pi}{c} R_{\Delta}(\tau) f_{c}\right)
$$

After range alignment and phase compensation, supposing there are $K$ scatters in the range cell which scatter $P$ is in, the signal in the range cell is

$$
S(f, \tau)=\sum_{k=1}^{K} \sigma_{k} T_{p} \exp \left(-j \frac{4 \pi}{c} R_{\Delta}(\tau) f_{c}\right)
$$

where $\sigma_{k}$ is reflection coefficient of the $k$-th scatter.

\section{NOVEL CS ISAR IMAGING ALGORITHM}

First, CS is briefly reviewed. Suppose $\Phi \in C^{N \times N}$ is basis space of signal $x \in C^{N}$, ie $x=\Phi s$, where $s$ is $K$ sparse coefficient vector, ie the number of non-zero coefficient satisfies $K \ll N$. Based on measurement matrix $\Psi \in C^{M \times N}(M<N), M$ dimension measurement $y$ can be obtained $y=\Psi x$. If $\Theta=\Psi \Phi$ satisfies restricted isometry property (RIP) condition, original signal $x$ can be reconstructed accurately with high probability based on observation $y$ by solving an optimization problem.

Then, ISAR imaging in cross-range is transformed into a CS problem. Formula (8) can be written in sparse representation model

$$
x=\Phi s
$$

where $x \in C^{N \times 1}$ is signal in one range cell, $\Phi \in C^{N \times N}$ represents Fourier basis matrix, $s \in C^{N \times 1}$ is $K$ sparse coefficient vector which indicates the distribution of scatters of targets, and $N$ is the total number of samples in cross-range. By employing measurement matrix $\Psi \in C^{M \times N}(M<N)$, signal is observed compressively

$$
y=\Psi x+n=\Theta s+n
$$

where $y$ is measurement, $\Theta=\Psi \Phi$, and $n$ is noise. Then $s$ can be solved by

$$
\min \|s\|_{0} \text { st }\|y-\Theta s\|_{2} \leq \varepsilon
$$

where $\|\cdot\|_{0}$ denotes $l_{0}$ norm, and $\varepsilon$ is the noise level.

As the above formula is a NP-hard non-convex optimization problem, it is usually transformed into a convex optimization problem based on $l_{1}$ norm. However studies show that only in very strict conditions are the results of optimization based on $l_{1}$ norm and $l_{0}$ norm equal. So in many cases, the method based on $l_{1}$ norm can't get good performance.

In 2013, Deng et al combined log-sum term into the framework of low-rank structure learning and demonstrated the log-sum heuristic recovery algorithm performs much better than the convex-norm-based method [9]. And Shen et al have provided a theoretical justification for adopting the log-sum as an alternative sparsityencouraging function [10]. Inspired by their work, log-sum minimization is employed here to improve imaging performance. Replacing $l_{0}$ norm in formula (11) with log-sum penalty function leads to

$$
\min \sum_{i=1}^{N} \log \left(\left|s_{i}\right|+\delta\right) \text { st }\|y-\Theta s\|_{2} \leq \varepsilon
$$

where $\delta>0$ is a very small constant to avoid negative infinity in log-sum penalty function. From a Bayesian perspective, there is intrinsic relevance between log-sum minimization and Sparse Bayesian Learning (SBL) [12]. In fact, log-sum minimization is a special case of $l_{p}$ norm as $p$ approaches zero on the left side [9]. And it is also shown in [10] that when $\delta \approx 0, \log$-sum penalty function is essentially the same as the $l_{0}$ norm. Therefore, compared with the $l_{1}$ norm and $l_{p}$ norm, log-sum minimization can approach $l_{0}$ norm more effectively.

However log-sum penalty function in formula (12) is a concave function. Fortunately, majorization-minimization (MM) algorithm, an effective strategy for solving non convex problems, can be employed to solve formula (12). MM algorithm proceeds in an iterative way. In this problem, non convex optimization problem is solved by a series of convex optimization problems, ie the following updating rule is obtained

$$
s^{(n+1)}=\operatorname{argmin} \sum_{i=1}^{N} \frac{\left|s_{i}\right|}{\left|s_{i}^{(n)}\right|+\delta} \mathrm{st}\|y-\Theta s\|_{2} \leq \varepsilon
$$




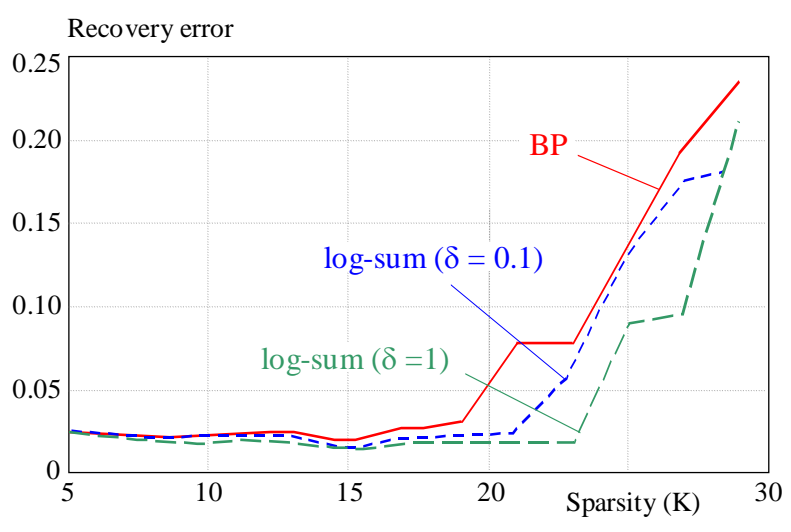

Fig. 1. Recovery errors of algorithms based on log-sum minimization and $l_{1}$ norm for different sparsity

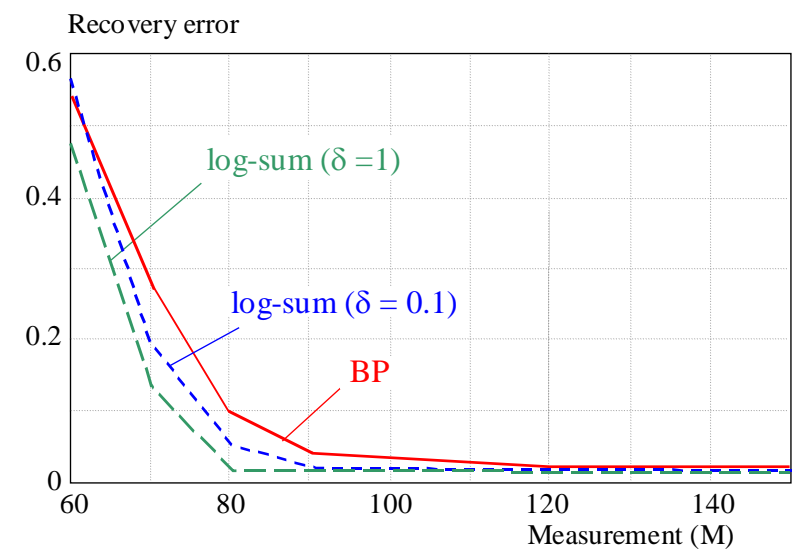

Fig. 2. Recovery error of the proposed algorithm and BP algorithm with different numbers of measurements

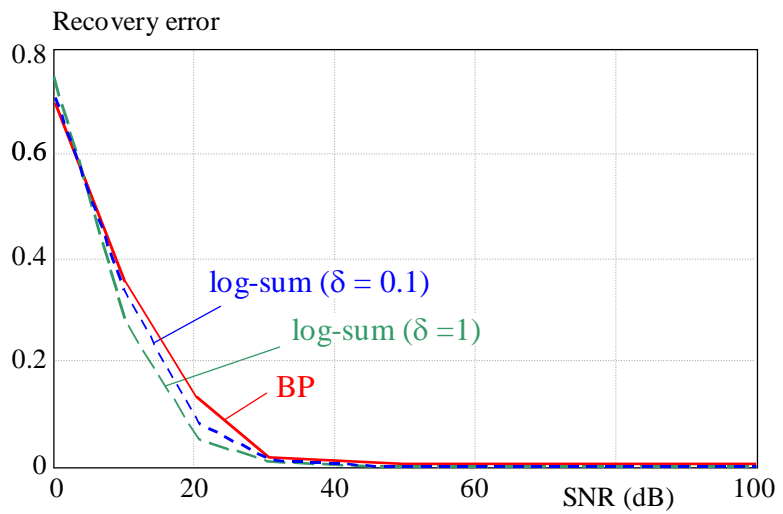

Fig. 3. Recovery error of the proposed algorithm and BP algorithm in different SNR

where $n$ represents the number of iterations.

Importantly, a new interpretation of the proposed algorithm is provided in the following. A novel reconstruction model deducted from Meridian prior is proposed [6]

$$
\min \sum_{i=1}^{N} \log \left(1+\frac{\left|s_{i}\right|}{2 \beta_{s}}\right) s t\|y-\Theta s\|_{2} \leq \varepsilon
$$

Where $\beta_{s}$ is the scaling parameter in Meridian prior. As $\beta_{s}$ is a constant, formula (14) can be written as

$$
\min \sum_{i=1}^{N} \log \left(2 \beta_{s}+\left|s_{i}\right|\right) \text { st }\|y-\Theta s\|_{2} \leq \varepsilon .
$$

If the scaling parameter $\beta_{s}$ is very small, let $2 \beta_{s}=\delta$, and formula (15) is equivalent to the log-sum minimization of (12). Consequently, sparsity enhancement evaluation via Lorentz curve and signal compressibility analysis in [6] also provide theoretical basis for log-sum minimization from another perspective. However, compared with the solution algorithm in [6], the proposed algorithm is much simpler and has lower computational cost.

\section{EXPERIMENTAL RESULTS AND DISCUSSION}

The effectiveness of the proposed algorithm has been verified by processing simulation data and real data.

First, the new algorithm is used in one dimensional real value sparse signals recovery to show log-sum penalty function superior to $l_{1}$ norm in CS.

Experiment 1: Performance of the proposed algorithm and $\mathrm{BP}$ algorithm based on $l_{1}$ norm in different sparsity conditions is compared. Sparse signals of size $N=100$ are used in experiments. The sparsity $K$ ranges from 5 to 30 . The amplitudes of non zero signal values obey normal distribution with mean 0 and variance 10 . Measurement matrix employs random Gaussian matrix of size $60 \times 100$. White Gaussian noise is added to the signal, which makes $\mathrm{SNR}=30 \mathrm{~dB}$. The values of $\delta$ in log-sum minimization are 0.1 and 1 , respectively. The average recovery errors of 50 experiments are shown in Fig. 1.

As shown in Fig. 1, the recovery errors of the proposed algorithm are lower than that of $\mathrm{BP}$ algorithm. What's more, the recovery error of BP algorithm starts to increase significantly from $K=15$, while the recovery error of the proposed algorithm begins to increase visibly from $K=21$. So compared with BP algorithm, the proposed algorithm can reconstruct signals in looser sparsity condition.

Experiment 2: Performance of the proposed algorithm and BP algorithm with different numbers of measurements is compared. Sparse signals of size $N=256$ with sparsity of $K=20$ are employed in experiments. The amplitudes of non zero signal values obey normal distribution with mean 0 and variance 10 . Measurement matrix is $M \times 256$ dimensional random Gaussian matrix, where $M$ ranges from 60 to 150 . White Gaussian noise is added to the signal, which makes SNR $=30 \mathrm{~dB}$. The values of $\delta$ in $\log$-sum minimization are 0.1 and 1 , respectively. Average recovery errors of 50 experiments are shown in Fig. 2.

It can be seen, on the whole, recovery errors of the proposed algorithm are lower than that of BP algorithm. The recovery errors of the proposed algorithm are close to 0 when $M \geq 90$, while the recovery errors of BP algorithm approach 0 when $M \geq 120$. Therefore, compared 

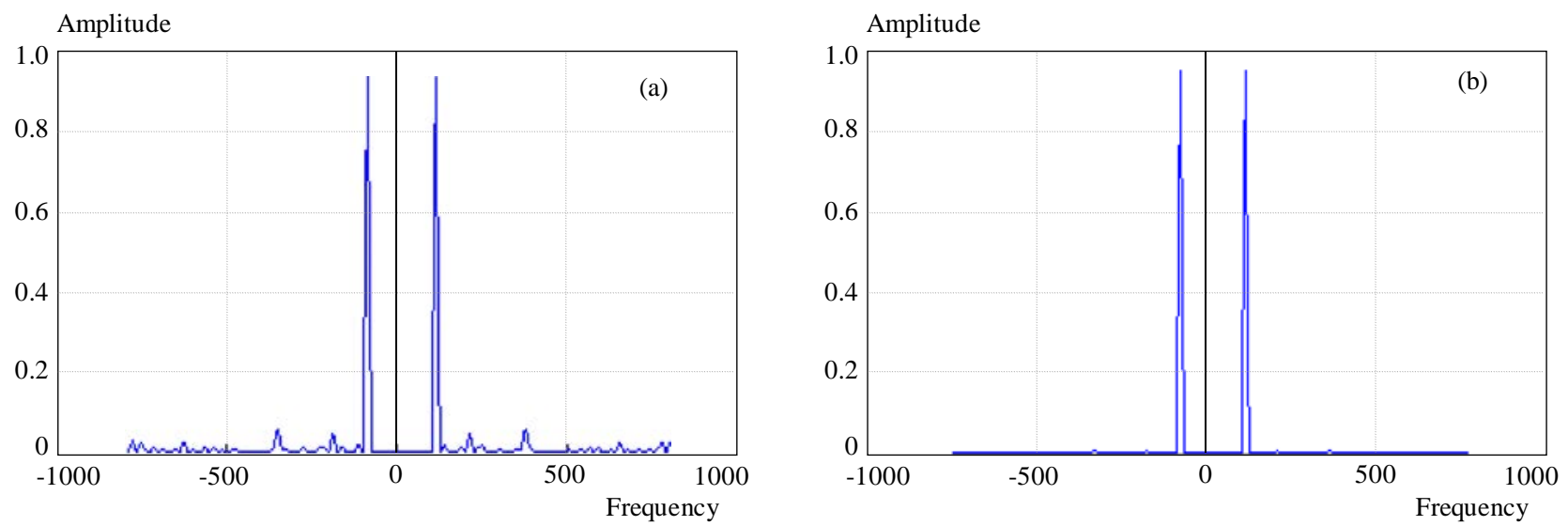

Fig. 4. Spectrum estimation of sinusoidal signal, (a) — based on BP algorithm, (b) — based on log-sum minimization
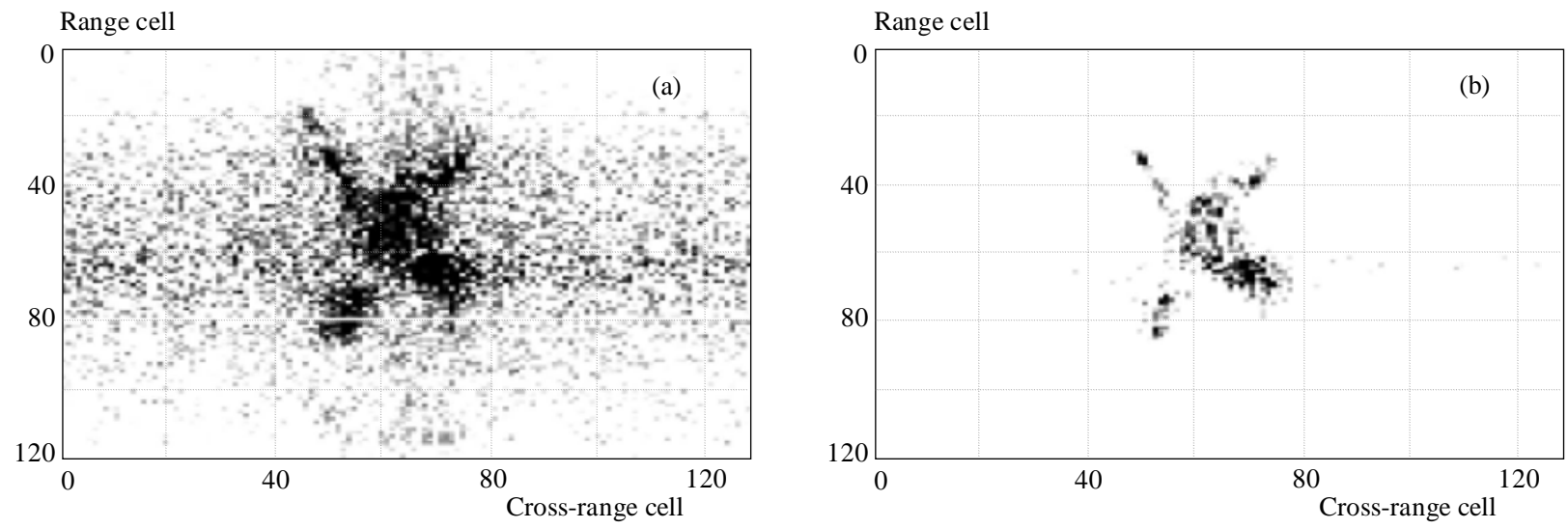

Fig. 5. CS ISAR imaging of real data, (a) — based on BP algorithm, (b) — based on log-sum minimization

Table 1. Parameters of radar

\begin{tabular}{cc}
\hline Parameter & Value \\
\hline Carrier frequency & $5.52 \mathrm{GHz}$ \\
Bandwidth & $400 \mathrm{MHz}$ \\
Pulse width & $25.6 \mu \mathrm{s}$ \\
Pulse repetition frequency & $400 \mathrm{~Hz}$ \\
Sampling frequency & $10 \mathrm{MHz}$ \\
\hline
\end{tabular}

with $\mathrm{BP}$ algorithm, the proposed algorithm can reconstruct signal with fewer measurements.

Experiment 3: The performance of the proposed algorithm and $\mathrm{BP}$ algorithm in different SNR is compared. The sparse signals in experiment 2 with parameter $M=90$ are employed here. SNR ranges from $0 \mathrm{~dB}$ to $100 \mathrm{~dB}$ and the values of $\delta$ in log-sum minimization are 0.1 and 1 . Average recovery errors of 50 experiments are shown in Fig. 3.

It can be seen from Fig. 3 , when $\mathrm{SNR} \geq 50 \mathrm{~dB}$, the recovery errors of the two algorithms approach 0 , ie they both get excellent results. When $5 \mathrm{~dB} \leq \mathrm{SNR}<50 \mathrm{~dB}$, the recovery errors of the proposed algorithm are less than that of BP algorithm. The recovery errors of BP algorithm are less than that of the proposed algorithm when $\mathrm{SNR}<5 \mathrm{~dB}$, but the recovery errors are very big, ie neither of the two algorithms can reconstruct signal here. In a word, the performance of the proposed algorithm is not worse than that of BP algorithm in different SNR when signals can be reconstructed successfully.

Second, the proposed algorithm is used in spectrum estimation of sinusoidal signal, which simulates the process of CS ISAR imaging in cross-range.

Experiment 4: The performance of the proposed algorithm and BP algorithm in spectrum estimation of sinusoidal signal is compared. A length of $N=128$ sinusoidal signal is used, where frequency $f_{0}=100 \mathrm{~Hz}$, amplitude is 2 , and sparsity $K=2$. Sparse basis is $128 \times 128$ dimensional discrete Fourier matrix and measurement matrix is $30 \times 128$ dimensional random Gaussian matrix. White Gauss noise is added to the signal, which makes $\mathrm{SNR}=15 \mathrm{~dB}$. The value of $\delta$ in log-sum minimization is 0.1. The estimated spectrum is shown in Fig. 4.

It can be seen from Fig. 4 that there is extra clutter in the spectrum obtained by BP algorithm, while the proposed algorithm has obtained clear spectrum. So in spectrum estimation of sinusoidal signal, the proposed algorithm has more advantages over BP algorithm.

Last, the proposed algorithm is applied into real data from ground-based ISAR radar to verify its feasibility in CS ISAR. 
Experiment 5: Imaging performance of the two algorithms for real ISAR data of Yark-42 aircraft is compared. Radar parameters are shown in Table 1. The data set consists of 128 pulses. Suppose due to system and maneuver limitations, there are only 64 pulses available in cross-range. To improve imaging resolution in crossrange, CS ISAR imaging algorithm is used. Sparse basis is $128 \times 128$ dimensional discrete Fourier matrix and measurement matrix is $64 \times 128$ dimensional random Gaussian matrix. Imaging results are shown in Fig. 5.

As shown in Fig. 5, there are many artificial points in Fig. 5(a), which almost overwhelm the strong scattering points of the target, so the imaging quality is not good. While a clear image of target has been obtained in Fig. 5(b), ie the strong scattering points of target are well recovered and there are not extra artificial points around the target, so the imaging quality is good. Therefore to real data, imaging performance of the proposed algorithm is superior to that of $\mathrm{BP}$ algorithm.

\section{CONCLUSION}

A new CS ISAR imaging algorithm based on log-sum minimization has been proposed in the paper. Compared with $\mathrm{BP}$ algorithm based on $l_{1}$ norm, it can not only reconstruct signals with fewer measurements or in looser sparsity conditions or with smaller recovery errors, but also estimate sinusoidal signal spectrum better. When applied into real ISAR data imaging, the proposed algorithm has obtained better imaging result than BP algorithm. So the new algorithm is a promising CS ISAR imaging method.

One scientific contribution of the paper is that a novel CS ISAR algorithm has been proposed, which is simple and has a good theoretical basis. At present, CS ISAR imaging performance is significantly dependent on the number of pulse, the sparsity of targets and the noise level. The proposed algorithm has better performance than the algorithm based on $l_{1}$ norm with decrease of the number of pulses and signal-to-noise ratio, and increase of the sparsity.

The other original contribution is that a novel interpretation of the proposed algorithm has been provided and a relation with the model based on Meridian norm has been built.

\section{Acknowledgments}

This work was supported by National Natural Science Foundation of China (grant No. 61301199, No .61378027), Research Fund for the Doctoral Program of Higher Education of China (grant No. 20122304120017), and the Fundamental Research Funds for the Central Universities (grant No. 2016B12114, No. 2016B02014).

\section{REFERENCES}

[1] ZHANG, L.-DUAN, J.-QIAO, Z. J.-XING, M. D.-BAO, Z. : Phase Adjustment and ISAR Imaging of Maneuvering Targets with Sparse Apertures, IEEE Transactions on Aerospace and Electronic System 50 No. 3 (2014), 1955-1973.

[2] XU, G.-XING, M. D.-XIA, X. G.-CHEN, Q. Q.-ZHANG, L.-BAO, Z.: High-Resolution Inverse Synthetic Aperture Radar Imaging and Scaling with Sparse Aperture, IEEE Journal of Selected Topics in Applied Earth Observations and Remote Sensing 8 No. 8 (2015), 4010-4027.

[3] DONOHO, D. L. : Compressed Sensing, IEEE Transactions on Information Theory $\mathbf{5 2}$ No. 4 (2006), 1289-1306.

[4] ZHANG, L.-XING, M. D.-QIU, C. W.-LI, J.-SHENG, J. L.-LI, Y. C.-BAO, Z. : Resolution Enhancement for Inversed Synthetic Aperture Radar Imaging under Low SNR via Improved Compressive Sensing, IEEE Transactions on Geoscience and Remote Sensing 48 No. 10 (2010), 3824-3838.

[5] FENG, C.-XIAO, L.-WEI, Z. H.: Compressive Sensing Inverse Synthetic Aperture Radar Imaging Based on Gini Index Regularization, International, Journal of Automation and Computing 11 No. 4 (2014), 441-448.

[6] ZHAO, G. H.-WANG, Z. Y.-WANG, Q.-SHI, G. M.SHEN, F. F.: Robust ISAR Imaging Based on Compressive Sensing from Noisy Measurements, Signal Processing 92 No. 1 (2012), 120-129.

[7] LI, S. D.-YANG, J.-MA, X. Y.: High Resolution ISAR Imaging Algorithm Based on Compressive Sensing (in Chinese), Journal on Communications 34 No. 9 (2013), 150-157.

[8] HYDER, M. M.-MAHATA, K. : Direction-of-Arrival Estimation Using a Mixed 12,0 Norm Approximation, IEEE Transactions on Signal Processing 58 No. 9 (2010), 4646-4655.

[9] DENG, Y.-DAI, Q. H.-LIU, R. S.-ZHANG, Z. K.-HU, S. Q.: Low-Rank Structure Learning via Nonconvex Heuristic Recovery, IEEE Transactions on Neural Networks and Learning Systems 24 No. 3 (2013), 383-396.

10] SHEN, Y. N.-FANG, J.-LI, H. B.: Exact Reconstruction Analysis of Log-Sum Minimization for Compressed Sensing, IEEE Signal Processing Letters 20 No. 12 (2013), 1223-1226.

[11] SRIPERUMBUDUR, B. K.-TORRES, D. A.-LANCKRIET, G. R. G. : A Majorization-Minimization Approach to the Sparse Generalized Eigenvalue Problem, Machine Learning 85 No. 1-2 (2011), 3-39.

12] WANG, F.-XIANG, X.-YI, K. C.-XIONG, L. : Sparse Signals Recovery Based on Latent Variable Bayesian Models (in Chinese), Journal of Electronics \& Information Technology $\mathbf{3 7}$ No. 1 (2015), 97-102.

Received 18 November 2015

Cheng Ping (lecturer) was born in Jiangsu, China, on July 2, 1976. She received Bachelor Degree and Master Degree in electronic engineering from Harbin Institute of Technology in 2000 and 2002 respectively, and $\mathrm{PhD}$ degree in the field of communication and information system in Harbin Institute of Technology in 2007. She has been working in Harbin Engineering University since 2007 and is with Hohai University now. Her research interest is compressed sensing, ISAR (inverse synthetic aperture radar) imaging. She has published nearly 30 journals and conference papers.

Zhao Jiaqun (associate professor) was born in Heilongjiang, China, in 1976. He received Master Degree in optics and $\mathrm{PhD}$ degree in physical electronics from Harbin Institute of Technology in 2003 and 2011, respectively. His research is mainly focused on solid state laser and nonlinear optical frequency conversion. 